\title{
The ExoMars Spectral Tool (ExoSpec): an image analysis tool for ExoMars 2020 PanCam imagery
}

Elyse J. Allender, Roger B. Stabbins, Matthew D. Gunn, Claire R. Cousins, Andrew J. Coates

Elyse J. Allender, Roger B. Stabbins, Matthew D. Gunn, Claire R. Cousins, Andrew J. Coates, "The ExoMars Spectral Tool (ExoSpec): an image analysis tool for ExoMars 2020 PanCam imagery," Proc. SPIE 10789, Image and Signal Processing for Remote Sensing XXIV, 107890I (9 October 2018); doi: $10.1117 / 12.2325659$

SPIE. Event: SPIE Remote Sensing, 2018, Berlin, Germany 


\title{
The ExoMars Spectral Tool (ExoSpec): an image analysis tool for ExoMars 2020 PanCam imagery
}

\author{
Elyse J. Allender, ${ }^{\mathrm{a},{ }^{*}}$, Roger B. Stabbins ${ }^{\mathrm{b}}$, Matthew D. Gunn ${ }^{\mathrm{c}}$, Claire R. Cousins ${ }^{\mathrm{a}}$, Andrew J. \\ Coates $^{\text {b }}$ \\ ${ }^{a}$ University of St Andrews, School of Earth and Environmental Sciences, Irvine Building, St Andrews, UK, KY16 9AL \\ ${ }^{\mathrm{b}}$ University College London, Mullard Space Science Laboratory, Holmbury Hill Rd, Dorking, UK, RH5 6NT \\ ${ }^{c}$ Aberystwyth University, Department of Physics, Penglais Campus, Aberystwyth, UK, SY23 3BZ
}

\begin{abstract}
The upcoming launch of the European Space Agency (ESA) ExoMars 2020 rover signals a need for an analysis tool to be created which can exploit the multi- and hyperspectral data that will be returned by its Panoramic Camera (PanCam), Infrared Spectrometer for Mars (ISEM), and Close-UP Imager (CLUPI) instruments. Data processed by this analysis tool will be invaluable in (i) characterising the geology local to the ExoMars rover, (ii) relating ground-based observations to orbital Compact Reconnaissance Imaging Spectrometer for Mars (CRISM) data, (iii) detecting evidence of past habitability on Mars, and (iv) identifying drilling locations. PanCam, ISEM, and CLUPI offer spectral analysis capabilities in both spatial (140-1310 microns/pixel at $2 \mathrm{~m}$ working distance) and spectral (440-3300 nm) dimensions. We have developed the ExoMars Spectral Tool (ExoSpec) which functions as a GUI-based extension to ENVI + IDL and performs steps from image import and compilation into ENVI .dat format, flat-fielding, radiometric correction, radiance-toreflectance $\left(\mathrm{R}^{*}\right)$ corrections using the in-scene Gretag MacBeth ColorChecker ${ }^{\mathrm{TM}}$, and calculation of spectral parameters. We demonstrate the functionality of ExoSpec at its current stage of development and illustrate its utility with results from field expeditions to Mars analogue terrains in: (i) geothermally altered basalts in Námafjall, Iceland, and (ii) layered alluvial plains deposits in Hanksville, USA, using ExoMars PanCam, ISEM, and CLUPI emulator instruments.
\end{abstract}

Keywords: European Space Agency, ESA, ExoMars, PanCam, ENVI, IDL, spectral, processing.

*Elyse J. Allender, ea63@st-andrews.ac.uk 


\section{Introduction}

Remote sensing is a widely used technique for mineral detection and characterisation in terrestrial and planetary applications at a variety of spectral and spatial scales. Orbital spectrometers collect regional scale imagery, while ground-based spectrometers collect local scale imagery. This local scale is particularly important for in-situ planetary exploration. In 2020, the European Space Agency (ESA) and Roscosmos will deliver the ExoMars rover and surface platform to the surface of Mars, whose communications with Earth will be supported by the already operational ExoMars Trace Orbiter (TGO). The ExoMars rover Pasteur payload ${ }^{1,2}$ contains a number of imaging instruments, including the Panoramic Camera (PanCam) ${ }^{3}$, the Infrared Spectrometer for ExoMars (ISEM) ${ }^{4}$, and the CLose UP Imager (CLUPI) ${ }^{5}$.

PanCam images downlinked from Mars will contain Digital Numbers (DN) representing each pixel. These DN require correction to radiance, and then reflectance, in order to represent meaningful surface information. At present, no custom software package exists to perform these steps for ExoMars 2020 PanCam and facilitate the manipulation and extraction of scientific information from these images. Furthermore, there is a scientific need to facilitate comparison with complementary datasets provided by ISEM and CLUPI. Having such a processing package is important because fast turnaround of imagery is required for rover operations, particularly strategic and tactical planning. Packages that are distributable to scientists and are easily added onto existing software play an important role in the pre-flight cross-calibration of instrument data as well as the scientific analysis of data returned from the ExoMars 2020 mission; which will be the primary purpose of ExoSpec, in collaboration with other designated PanCam software, such as Pro3D'.

We introduce ExoSpec (the ExoMars Spectral Tool), a processing package which runs as an extension to ENVI + IDL 8.6 and is currently used to process Aberystwyth University PanCam Emulator (AUPE3) data collected during Mars analogue studies, in preparation for ExoMars. Future iterations of this tool will be able to process ExoMars PanCam imagery in addition to AUPE3 imagery to produce interpretable image products, containing over 1 million spectral signatures, which provide both regional and local scale context for other instruments.

ExoSpec's heritage stems from processing procedures applied to the NASA Imager for Mars Pathfinder (IMP) 7,8, Mars Exploration Rover (MER) Pancams 9 , Mars Science Laboratory (MSL) Mastcam $^{10}$, AUPE2 ${ }^{11}$, and the Mars Reconnaissance Orbiter's (MRO) Compact Reconnaissance Imaging Spectrometer for Mars (CRISM) ${ }^{12}$ instrument. Processing pipelines, like the one developed for the IMP by the Multimission Image Processing Laboratory (MIPL) at the Jet Propulsion Laboratory (JPL), perform image calibration and mosaicking in preparation for science products such as slope maps, roughness maps, and reachability maps to be created. ${ }^{13,14}$ Additional science products, such as spectral parameter maps and false colour mosaics, have also been generated for a variety of instruments including MER Pancam, MSL MastCam, AUPE2, and CRISM. ${ }^{11,15-19}$ In particular, the CRISM Analysis Toolkit (CAT) ${ }^{20}$ served as the inspiration for ExoSpec due to its ease of installation, and userfriendly interface. As no pipeline currently exists for ExoMars PanCam that incorporates the entire workflow from image ingestion and pre-processing to science product generation we seek to fill this gap with ExoSpec; making the entire process of generating ExoMars PanCam multispectral science products accessible and user-friendly.

At its current stage of development the main function of ExoSpec is to generate relative reflectance images from raw data so that an assessment of spectral variability within a scene can be made. Spectral 
parameter maps are also generated, to provide an overview of regional and local geological context. At its final stage of development, the ultimate goal of ExoSpec is to provide the tools to perform detailed mineral characterisation of an ExoMars PanCam scene utilising a combination of multi-scale data provided by the PanCam, ISEM, and CLUPI instruments. With this information, targets of interest for science return can then be planned in order to aid in the search for biosignatures and contribute to in-depth scientific investigations relating to the key goals of the ExoMars 2020 mission $^{3}$.

For the development of ExoSpec, we took PanCam, ISEM, and CLUPI emulators (AUPE3, ISEM$\mathrm{E}$, and CLUPI-E respectively) to analogue terrains in Iceland and the USA and collected spectral data to be processed and analysed. The structure of this paper is as follows: section 2 provides background to the Pancam, ISEM, and CLUPI instruments and their emulators. Section three details the workflow of ExoSpec. Section 4 is concerned with the results from recent field trials. Each analogue field site is introduced and examples of unprocessed and processed image data are presented. Section 5 discusses future developments for the pipeline, and section 6 concludes the paper.

\section{ExoMars 2020 Imaging Instruments}

The remote sensing instruments onboard the ExoMars 2020 rover provide multi-scale, multi- and hyper-spectral information within a single scene. Cross-calibration of this data pre-launch will aid in the interpretation of mineralogy and potential biosignature locations in the primary science phase of the mission, thus, the final iteration of ExoSpec will enable data from all three instruments in this section to be compared and employed in combination for geological analysis.

\subsection{Panoramic Camera (PanCam)}

To provide the ExoMars rover with local scale morphological and mineralogical context the Panoramic Camera (PanCam $)^{3}$ has been incorporated on the mast as the 'eyes' of the rover. PanCam is composed of two wide angle cameras (WAC) which together are able to image a $38.3^{\circ}$ field of view (FOV) across a 440-1000 nm spectral range. The filter wheel within each camera is comprised of three broadband colour filters, six narrowband geology filters, and two solar filters (Table 1). For AUPE3, in place of these solar filters in positions 10 and 11 there is a luminance filter covering the visible portion of the spectrum, and an empty filter slot for panchromatic imaging. ${ }^{11}$ Details of the filter wheels in Left WAC (LWAC) and Right WAC (RWAC) cameras for the PanCam emulator (AUPE3) we use in this study versus the ExoMars PanCam are provided in Table 1. If an LWAC or RWAC image is captured using all filters in the wheel, the result is an 11-band multispectral file. If only geology filters are captured in either LWAC or RWAC, the result is a 6-band multispectral file.

ExoMars PanCam's heritage comes from the Beagle 2 Stereo Camera System ${ }^{22}$ which was part of ESAs Mars Express mission, and from the National Aeronautics and Space Administration's (NASA) MER Pancams ${ }^{23}$ onboard the Spirit and Opportunity rovers. While the spectral filters differ between ExoMars and MER Pancam iterations, existing data exists about image pre-processing, processing, and mineralogical interpretation of this data. The Aberystwyth University Pancam Emulator (AUPE3) has been built with off-the-shelf components to replicate ExoMars PanCam specifications and is used in the field to test the imaging capabilities of the instrument in Mars analogue terrains. ${ }^{11}$ To correct

raw image data to relative reflectance, the Gretag Macbeth Colorchecker ${ }^{\mathrm{TM}}$ is used in tandem with this instrument to emulate the calibration target to be positioned on ExoMars' deck. ${ }^{24}$ 
Table 1 Filter wheel specification of AUPE3 and ExoMars PanCam., ${ }^{3,21}$ WAC filter bandpasses are given as Full Width at Half Maximum (FWHM) values. Units are nanometres.

\begin{tabular}{|c|c|c|c|c|c|c|c|}
\hline \multirow[b]{3}{*}{ Filter \# } & \multirow[b]{3}{*}{ Filter } & \multicolumn{2}{|l|}{ AUPE3 } & \multirow[b]{3}{*}{ Filter \# } & \multirow[b]{3}{*}{ Filter } & \multicolumn{2}{|l|}{ ExoMars PanCam } \\
\hline & & LWAC & RWAC & & & LWAC & RWAC \\
\hline & & Centre wavelength ( $F W H M)$ & Centre wavelength ( $F W H M$ ) & & & Centre wavelength ( $F W H M)$ & Centre wavelength $(F W H M)$ \\
\hline 1 & Blue Broadband & $440(120)$ & $440(120)$ & 1 & Blue Broadband & $440(120)$ & $440(120)$ \\
\hline 2 & Green Broadband & $540(80)$ & $540(80)$ & 2 & Green Broadband & $540(80)$ & $540(80)$ \\
\hline 3 & Red Broadband & $640(100)$ & $640(100)$ & 3 & Red Broadband & $640(100)$ & $640(100)$ \\
\hline 4 & Geology 1 & $438(24)$ & $740(13)$ & 4 & Geology 1 & $440(25)$ & $740(15)$ \\
\hline 5 & Geology 2 & $500(24)$ & $780(10)$ & 5 & Geology 2 & $500(20)$ & $780(20)$ \\
\hline 6 & Geology 3 & $532(10)$ & $832(37)$ & 6 & Geology 3 & $530(15)$ & $840(25)$ \\
\hline 7 & Geology 4 & $568(10)$ & $900(50)$ & 7 & Geology 4 & $570(12)$ & $900(30)$ \\
\hline 8 & Geology 5 & $610(10)$ & $950(50)$ & 8 & Geology 5 & $610(10)$ & $950(50)$ \\
\hline 9 & Geology 6 & $671(10)$ & $1000(50)$ & 9 & Geology 6 & $670(12)$ & $1000(50)$ \\
\hline 10 & Visible & $545(290)$ & $545(290)$ & 10 & Solar 1 & $925(5)$ & $450(5)$ \\
\hline 11 & Empty & Panchromatic & Panchromatic & 11 & Solar 2 & $935(5)$ & $670(5)$ \\
\hline
\end{tabular}

\subsection{Infrared Spectrometer for ExoMars (ISEM)}

Mounted below PanCam on the rover mast is the Infrared Spectrometer for ExoMars (ISEM) ${ }^{4}$. This instrument provides point spectra for a $1^{\circ} \mathrm{FOV}$ within the larger PanCam FOV, and the $4^{\circ} \mathrm{FOV}$ of the High Resolution Camera (HRC) ${ }^{3}$ which is mounted alongside PanCam on the optical bench. ISEM uses an acousto-optic tunable filter (AOTF) to cover the spectral range from 1150-3300 nm at variable resolution. It is projected that one ISEM spectrum will be captured per HRC image. Targeting of HRC and ISEM will be driven by the discovery of mineralogical and morphological features of interest observed in PanCam imagery. Combining all of these instruments allows detailed analysis of diagnostic mineral features in the VNIR to SWIR wavelengths. For the ISEM emulator, ISEM-E, we use a field spectrometer (Spectral Evolution RS-3500) with a spectral range of 350-2500 nm. While ISEM will not share any overlapping bands with PanCam, the overlap of ISEM-E and AUPE3 allows for spectral comparison between these two instruments during analogue field trials which will provide valuable information to relate their results.

\subsection{Close Up Imager (CLUPI)}

Mounted on the drill of ExoMars 2020 is the CLose-UP Imager (CLUPI) ${ }^{5}$. CLUPI is a high-resolution, colour camera system designed to mimic what a geologist would observe in the field through a hand lens, providing images of minerals within their original context before they are powdered for geochemical analyses. Such high-resolution data will facilitate the search for morphological features that may indicate biosignatures on outcrops and drill cores. When at a distance of $10 \mathrm{~cm}$ from a target, CLUPI provides 7 micron/pixel resolution with a FOV of $1.9 \times 1.3 \mathrm{~cm}$. The CLUPI emulator, CLUPI-E, is a Digital SLR with a macro lens which replicates CLUPI imaging capabilities.

\section{ExoMars Spectral Tool (ExoSpec)}

\subsection{Installation}

The minimum requirement for installation of ExoSpec is ENVI 8.1. The current version runs on a Windows system, but is also compatible with Apple distributions. To install, a user can simply copy the ExoSpec .sav file into their Harris/ENVIxx/extensions folder, where ' $x x^{\prime}$ ' represents the user-specific ENVI version. The next time ENVI is started, ExoSpec will appear as an option under the 'Display' dropdown menu. At its current stage of development, several camera options are visible once ExoSpec 
is selected. These are HRC, AUPE3, and PanCam. At present, only AUPE3 options are selectable, and separate, camera specific, processing paths exist for AUPE3 LWAC and AUPE3 RWAC.

\subsection{Processing workflow}

ExoSpec's processing routines will continue to evolve until the launch of the ExoMars 2020 mission to incorporate analyses for cross-calibration with ISEM and CLUPI and establish multispectral summary products relevant to specific geological/mineralogical features. At it's current stage of development, ExoSpec performs all pre-processing steps from camera radiometric calibration, radiance to reflectance correction, image masking, and band depth/band ratio product generation for AUPE3 images, as seen in Fig. 1. Each step in the flowchart will be explained in detail in this section. Image decompression and dark/bias subtraction steps are not implemented within ExoSpec because they will be performed at the Rover Operations Control Centre (ROCC) as images are downlinked. 'Flat-field correction' and 'conversion to radiance' steps will also be performed at the ROCC after ExoMars image downlink, but ExoSpec will continue to implement these steps for the AUPE3 processing stream.

All ExoSpec's user functions operate via a Graphical User Interface (GUI), so the user need only select directories and files they wish to operate within. In order to successfully calibrate a scene to reflectance, the first image a user processes in that scene must contain the calibration target, the reason for this will become apparent in section 3.2.4, but essentially, in the case of ExoMars, neartime observations of the calibration target will be used to derive parameters to characterise the lighting environment for each image in which the calibration target is not visible.

\subsubsection{Image Ingestion}

To begin processing, a user must first decide whether to process images from the LWAC or RWAC. To create an 11-band multispectral image file for the chosen camera (containing all filters in a single column of Table 1), the user must point ExoSpec toward the directory containing all input files obtained from AUPE3. These files are in .png format and contain metadata such as filter names, band centres and bandpasses, wavelength units, exposure times, camera gain values, and the camera name. A directory for image output also must be selected or optionally created. Once these directories are set, filter files 1 to 11 can be selected in one import step by holding the CTRL key while individual files are selected.

During file import, image metadata is read from each individual filter file and combined into the new multispectral image header. It is recommended that all 11 filter images are selected to construct the initial multispectral image. Filters 4 to 9 (the geology filters) may be constructed to form a 6 band multispectral image if desired, however, a true colour RGB representation of the image will not be able to be viewed. At this stage, pixel units are in Digital Numbers (DN).

\subsubsection{Flat Fielding}

In order to remove pixel-to-pixel non-uniformities for each camera flat-fielding must be performed. These non-uniformities are typically caused by intrinsic responsivity variations among individual pixels on the detector, or by induced responsivity variations in the optics, individual filters, or outward variations such as dust specks on the lens. ${ }^{10}$ All flat fields for AUPE3 used in this report were measured at Aberystwyth University using an integrating sphere; each flat field image is the average of 30 


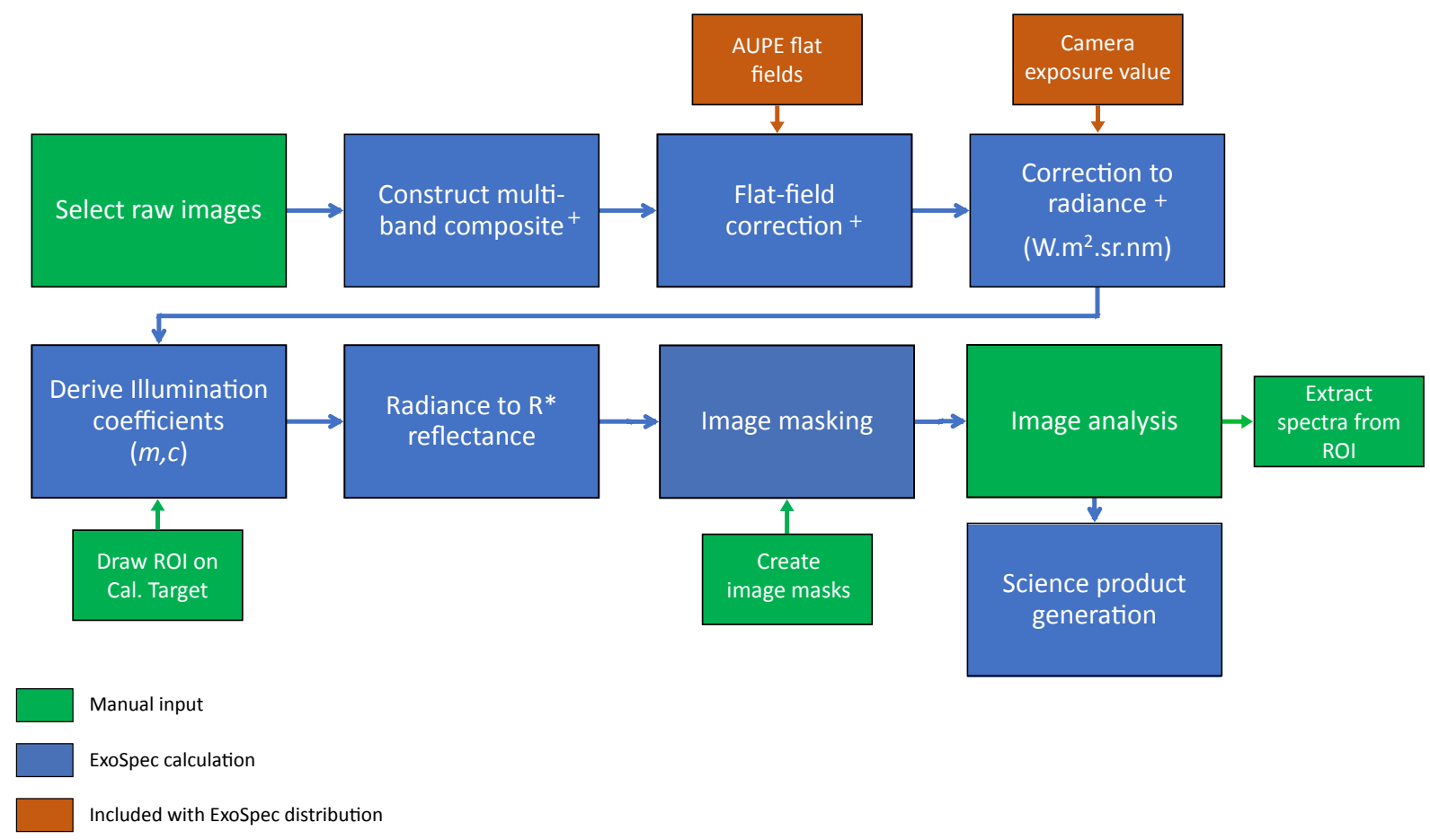

Fig 1 Flowchart of current ExoSpec processing routines. Steps marked with a cross will be performed at the ROCC for ExoMars and will not be part of the ExoSpec PanCam processing stream, however, these steps will be maintained for the AUPE3 processing stream.

individual observations, and there is one flat field image per filter. Flat-field images for AUPE3 are currently included in the ExoSpec distribution.

To perform the flat field correction ExoSpec normalises each flat field (so that each has a mean = 1). Each of the 11-bands in the multispectral image are then corrected by dividing each band by its normalised flat-field image. At this stage, pixel units remain in Digital Numbers (DN).

\subsubsection{Conversion to Radiance}

To convert the now flat-fielded multiband image into a calibrated radiance image (W.m ${ }^{2}$.sr.nm) from which true colour data products can be derived or spectra extracted for direct comparison with ISEM or laboratory spectra, the following equation (adapted from Ref. 9) is applied:

$$
R C_{\lambda}=K(T) * D N_{p s},
$$

where $\lambda$ is the filter number, $K(T)$ is a radiance conversion coefficient which is dependent on CCD temperature $T$ of the camera, and $D N_{\mathrm{ps}}=D N / e_{\lambda}$, where $e_{\lambda}$ is the exposure time for filter $\lambda$.

We have quantified $K(T)$ for AUPE3 via a gain value for each filter. Its value, along with the exposure time $(e)$ which converts the pixel units into DNs per second, is read directly from the image header metadata. We don't have temperature calibrated coefficients or a CCD thermometer for AUPE3, however, we expect fairly stable thermal conditions on Earth. For ExoMars PanCam, as with MER 


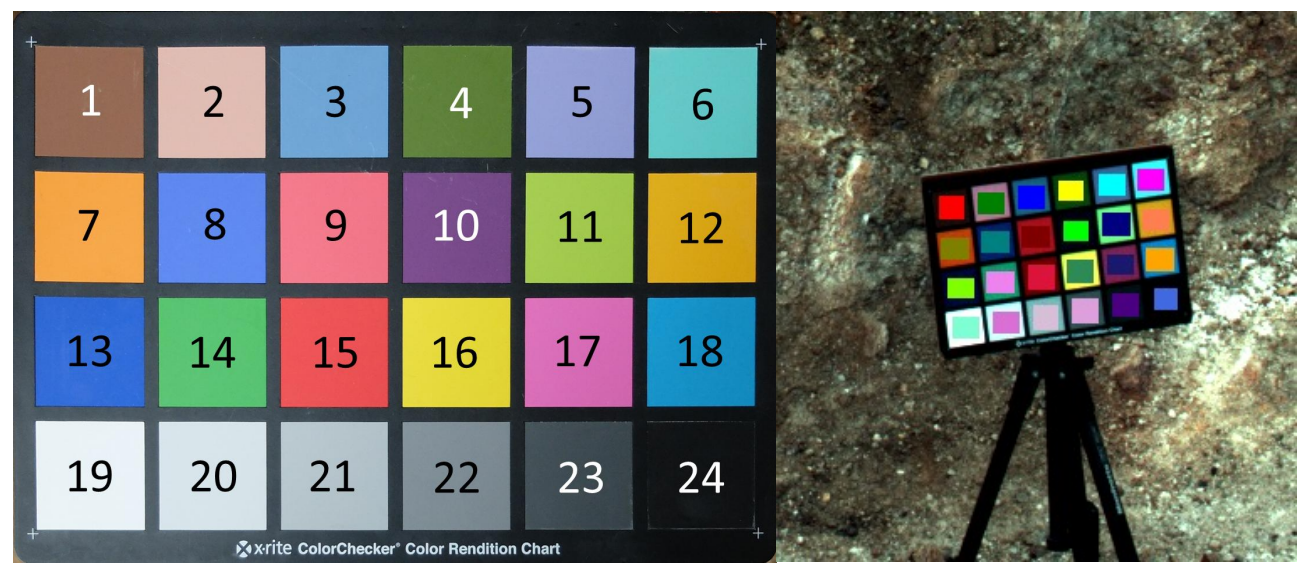

Fig 2 Left: Image ROIs must be created in the order shown above in order to be correctly read into ExoSpec. Right: An example of ROIs selected and overlaid on the calibration target in an LWAC scene.

Pancam, larger temperature changes are expected and as such more rigorous values for this radiometric conversion coefficient must be calculated, using the methods provided in Ref. 25.

The output from this step is a calibrated radiance image from which illumination coefficients may be calculated for correction to relative reflectance $\left(\mathrm{R}^{*}\right)$ using a calibration target. All intrinsic artefacts produced by the camera system have been corrected at this stage. Pixel units are now in W.m ${ }^{2}$.sr.nm.

\subsubsection{Calculation of Illumination Coefficients}

To correct images to reflectance, the illumination level at each wavelength of the image must be determined, thus, illumination coefficients must be obtained for input into Eq. (2). This requires that the user create regions of interest (ROI) over each coloured square of the calibration target (for this study, the Gretag MacBeth ColorChecker ${ }^{\mathrm{TM}}$ ) in the scene being analysed (Fig. 2). This procedure need only be performed twice per scene, once for each of the two LWAC and RWAC images that contain the calibration target. ROIs must be selected in the order specified in Fig. 2.

Once all ROIs have been created, ExoSpec calculates the average radiance for each individual ROI, and plots these relative to their reflectance values which were pre-measured at Aberystwyth University using a handheld spectrometer, and an integrating sphere (Fig. 4). One of these plots is produced per filter. By fitting a straight line through these 24 points using the weighted least-squares fitting algorithm from Ref. 26 an estimate of the illumination coefficients $m$ and $c$ are obtained for each filter using Eqs. (3) and (5). These coefficients are applied to all images in a scene which do not contain the calibration target to perform the subsequent correction from radiance to $\mathrm{R}^{*}$ reflectance in section 3.2.5.

$$
R^{*}=\frac{S-c}{m},
$$

where $R^{*}$ is the relative reflectance of the scene at each filter wavelength, $S$ is the average signal from each ROI, $m$ is the gradient of the line obtained from a weighted least squares fit, and $c$ is its intercept, 
calculated as in Eq. (3) to (5):

$$
\Delta=\sum_{i} \frac{1}{\sigma_{i}^{2}} \sum_{i} \frac{\rho_{i}^{2}}{\sigma_{i}^{2}}-\left(\sum_{i} \frac{\rho_{i}^{2}}{\sigma_{i}^{2}}\right)^{2},
$$

where $\sigma_{i}$ is the standard deviation of each ROI $i, \rho_{i}$ is the lab measured reflectance of each calibration target square $i, S_{i}$ is the average signal in W.m².sr.nm of each ROI $i$. Note that ROIs with larger uncertainty contribute less to the calculation of the illumination coefficients.

$$
m=\frac{\sum_{i} \frac{1}{\sigma_{i}^{2}} \sum_{i} \frac{\rho_{i} S_{i}}{\sigma_{i}^{2}}-\sum_{i} \frac{\rho_{i}}{\sigma_{i}^{2}} \sum_{i} \frac{S_{i}}{\sigma_{i}^{2}}}{\Delta}
$$

where $\sigma_{i}$ is the standard deviation of each ROI $i, \rho_{i}$ is the lab measured reflectance of each calibration target square $i, S_{i}$ is the average signal in W.m².sr.nm of each ROI $i$.

$$
c=\frac{\sum_{i} \frac{\rho_{i}^{2}}{\sigma_{i}^{2}} \sum_{i} \frac{S_{i}}{\sigma_{i}^{2}}-\sum_{i} \frac{\rho_{i}}{\sigma_{i}^{2}} \sum_{i} \frac{\rho_{i} S_{i}}{\sigma_{i}^{2}}}{\Delta}
$$

where $\sigma_{i}$ is the standard deviation of each ROI $i, \rho_{i}$ is the lab measured reflectance of each calibration target square $i, S_{i}$ is the average signal in W.m².sr.nm of each ROI $i$.

\subsubsection{Radiance to Reflectance $\left(R^{*}\right)$}

$\mathrm{R}^{*}$ was defined by Ref. 27 as "the brightness of the surface divided by the brightness of a radiometric calibration target scaled to its equivalent Lambertian reflectance". In simpler terms, it is the ratio of the reflectance of a surface to that of a perfectly diffuse surface under the same conditions of illumination and measurement. This is also known as "relative reflectance" because all reflectance within the scene is scaled relative to a target with a known reflectance value.

As mentioned in section 3.2.4 for the calibration of AUPE3 images to $\mathrm{R}^{*}$ reflectance, we use a Gretag MacBeth ColorChecker ${ }^{\mathrm{TM}}$ (Fig. 2). Images calibrated using such a calibration target may also be considered partially atmospherically corrected as observations of the calibration target include the average diffuse sky illumination component of the scene radiance. ${ }^{25}$

ExoSpec converts the radiance values for each filter obtained in section 3.2 .3 to $\mathrm{R}^{*}$ reflectance using the $\Delta, m$ and $c$ values derived in the previous section (3.2.4) using Eq. (2). Uncertainties for these illumination coefficients are calculated using Eq. (6) and (7), also from Ref. 26.

$$
\sigma_{m}=\sqrt{\frac{\sum_{i} \frac{1}{\sigma_{i}^{2}}}{\Delta}},
$$

where $\sigma_{i}$ is the standard deviation of each ROI $i$, and $\Delta$ is the weighting denominator from Eq. (3).

$$
\sigma_{c}=\sqrt{\frac{\sum_{i} \frac{\rho_{i}^{2}}{\sigma_{i}^{2}}}{\Delta}},
$$

where $\sigma_{i}$ is the standard deviation of each ROI $i, \rho_{i}$ is the lab measured reflectance of each calibration target square $i$, and $\Delta$ is the weighting denominator from Eq. (3). 


\subsubsection{Image Masking}

After an image has been converted to $\mathrm{R}^{*}$ reflectance, image masking is an optional step able to be performed if images contain extensive amounts of sky, vegetation, or shadow which a user may want to disregard from analysis before computing further science products. The user may select either to mask only one of these properties, or all. Masking these values before calculating science products will maximise their output range, highlighting small changes in mineralogy rather than using the majority of the available 0-255 range to represent small changes in sky, shadows, or vegetation.

Regions of sky or vegetation can be masked by creating a ROI mask within ENVI. The vignetting at the edges of the geology filters can also be masked using a ROI and processed as a "sky mask".

"Shadow masks" are created by specifying maximum and minimum range values within an image. When creating a "shadow mask" for AUPE3 images, a good starting point to determine a useful "shadow" value is the black calibration target square. Pixels with this value or below should be masked. Pixels with $\mathrm{R}^{*}$ values greater than 1 (typically occurring within the vignetted edge regions of the geology filters) can also be masked using this method.

These created masks can then be applied using the masking option within the ExoSpec Mask menu.

\subsubsection{Science Product Generation}

Perhaps the most defining feature of the present iteration of ExoSpec is the creation of spectral parameters, such as band depth and band ratio products, which capture the shape of the spectrum and aid in visualisation of regional and local mineralogy and subtle compositional differences imparted by lithology, weathering state, and dust cover.

Typically, spectral parameters are created to highlight areas of scientific interest in a scene. They have been used successfully at multiple scales for MER Pancam, ${ }^{16}$ and CRISM. ${ }^{17,19}$ For ExoMars, they will be used to refine potential locations for further investigation with ISEM and CLUPI and enable in-depth geological characterisation to be carried out in corroboration with structural analysis performed by Pro3D. ${ }^{6}$

For analogue testing with AUPE3, formulae from Refs. 16 and 11 have been incorporated into ExoSpec (Table 2). Depending on the camera used (LWAC or RWAC) all available summary parameters are automatically generated for an image once this menu option is selected. More specialised products will be developed as we continue to test ExoSpec with analogue field data, and, in future releases of ExoSpec, optimal combinations of these parameters will be determined, and will be calculated depending on the filter subset used for image capture.

\section{Results}

In this section we show results from fieldwork undertaken in the geothermal area of Námafjall, Iceland and in Hanksville, Utah, USA. For data collection we use AUPE3, ISEM-E, and CLUPI-E. Here we will show only the fieldwork location, and post-processed LWAC images, as each image does not significantly change visually during ExoSpec processing until spectral parameters are generated. To conserve space, processing outcomes, such as illumination coefficient graphs (Fig. 4) and ROI observed vs measured plots (Fig. 5) will be shown only for the Icelandic site. 
Table 2 Science products (spectral parameters) calculated for AUPE3 within ExoSpec.

\begin{tabular}{|c|c|c|}
\hline Name & Description & Rationale \\
\hline \multicolumn{3}{|l|}{ LWAC } \\
\hline S438_671 & {$[(\mathrm{R} 671-\mathrm{R} 438) /(671-438)]$} & Related to degree of oxidation \\
\hline BD532 & {$[1-(\mathrm{R} 532 /((0.53 * \mathrm{R} 500)+(0.47 * \mathrm{R} 568)))]$} & Identifies ferric minerals, particularly hematite, and is related to degree of oxidation \\
\hline BD610 & {$[1-(\mathrm{R} 610 /((0.6 * \mathrm{R} 568)+(0.4 * \mathrm{R} 671)))]$} & Can indicate goethite development and can be influenced by olivine and pyroxene \\
\hline S532_610 & {$[(\mathrm{R} 610-\mathrm{R} 532) /(610-532)]$} & Ferric minerals and dust \\
\hline R671_438 & [R671/R438] & Ferric minerals and dust \\
\hline \multicolumn{3}{|l|}{ RWAC } \\
\hline R740_1000 & [(R740/R1000)] & Ferrous minerals \\
\hline S740_1000 & {$[(\mathrm{R} 1000-\mathrm{R} 740) /(1000-740)]$} & Strength and position of NIR absorption linked to ferrous minerals \\
\hline BD900 & {$[1-(\mathrm{R} 900 /((0.455 * \mathrm{R} 840)+(0.545 * \mathrm{R} 950)))]$} & Strength of NIR absorption, related to ferric minerals \\
\hline S900_1000 & {$[(\mathrm{R} 1000-\mathrm{R} 900) /(1000-900)]$} & Related to detection of hydrous minerals \\
\hline BD950 & {$[1-(\mathrm{R} 950 /((0.5 * \mathrm{R} 900)+(0.5 * \mathrm{R} 1000)))]$} & Related to hydrous minerals, some clays, and silicates \\
\hline S950_1000 & {$[(\mathrm{R} 1000-\mathrm{R} 950) /(1000-950)]$} & Linked to detection of hydrous minerals \\
\hline
\end{tabular}

\subsection{Námafjall, Iceland}

Námafjall is a geothermal field in the central northern region of Iceland $\left(65.6382^{\circ} \mathrm{N}, 16.8197^{\circ} \mathrm{W}\right)$. We focus on Námafjall ridge, which is located at the centre of the geothermal field. At the base of the ridge are both active and extinct fumeroles, some of which can be seen in Fig. 3. At the top of the ridge are pillow basalts, at various stages of weathering and hydrothermal alteration. In this study, we show results from the altered pillow basalt outcrop - this is late stage alteration of pillow basalt containing gypsum veins (Fig. 6).

The altered pillow basalt outcrop was covered by a mosaic of three AUPE3 images. Focussing first on processing images from the LWAC, we located the image ID containing the calibration target and selected the 11 filter images with this ID and matching bracketing values as our first input. ExoSpec then combined these 11 individual .png files into an 11-band multispectral image file. Flat field correction was performed, along with radiometric correction, to produce radiance images.

At this point, ROIs were manually created for each coloured square of the calibration target, and illumination coefficients were automatically calculated by ExoSpec, resulting in the output in Fig. 4. The uncertainty of each of these coefficients is also automatically calculated at this point, and can be displayed to the user in ENVI. The radiance to $\mathrm{R}^{*}$ reflectance correction was then able to be performed, resulting in 24 plots being produced (one for each ROI on the calibration target) to illustrate the "goodness of fit" between observed image reflectance $\left(\mathrm{R}^{*}\right)$ and their lab measured reflectance. A subset of these output plots is shown in Fig. 5.

Vignetting at the edge of the newly produced $\mathrm{R}^{*}$ image was then masked along with heavily shadowed regions. LWAC spectral parameters were then produced using the masked $\mathrm{R} *$ image as input. In order to extract and examine spectra from the masked image, regions of interest were manually created over areas which appeared spectrally interesting within the spectral parameter image (Fig. 6); LWAC spectra were then extracted from these locations. This process was then repeated for the RWAC image containing the calibration target, after which spectra extracted from the regions of interest in each camera were merged to form a 12 filter spectral signature (consisting of only the geology filters from Table 1) for analysis and comparison with ISEM-E spectra (Fig. 7). All images in this set were then processed in the same manner, however, the 'calculation of illumination coefficients' step was omitted in subsequent processing as this need only be performed on images containing the calibration target.

From Fig. 7 it can be seen that the AUPE3 spectra extracted from this image match well to the spectra collected with ISEM-E, with the exception of a small artifact around $780 \mathrm{~nm}$ in the 'altered light material' ROI. 


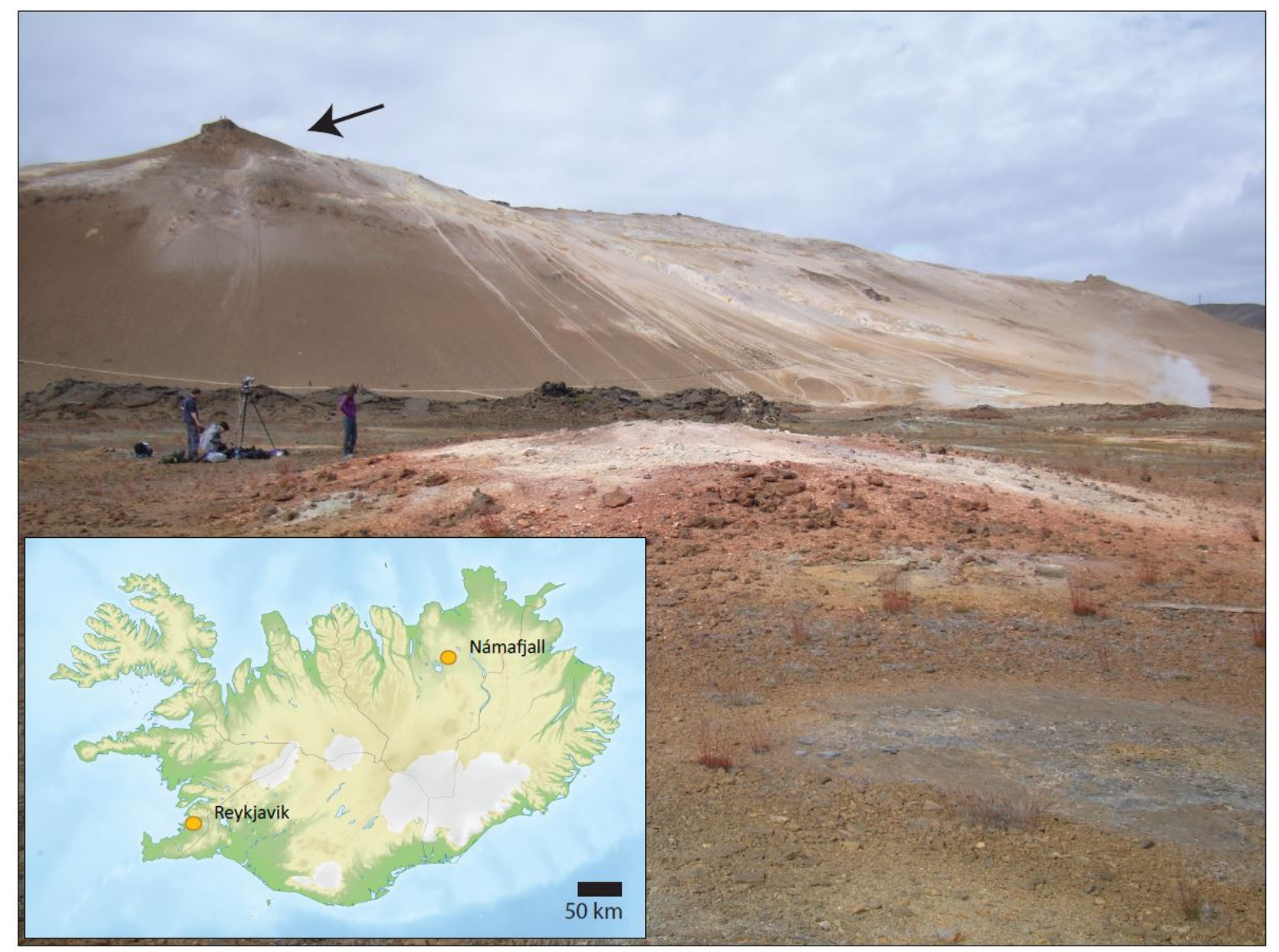

Fig 3 The inset image image shows the location of Námafjall geothermal field within Iceland. The main image was captured from within the geothermal field looking west toward Námafjall ridge. The pillow basalt being studied is located at the summit of the ridge, shown by the arrow. 
LWAC
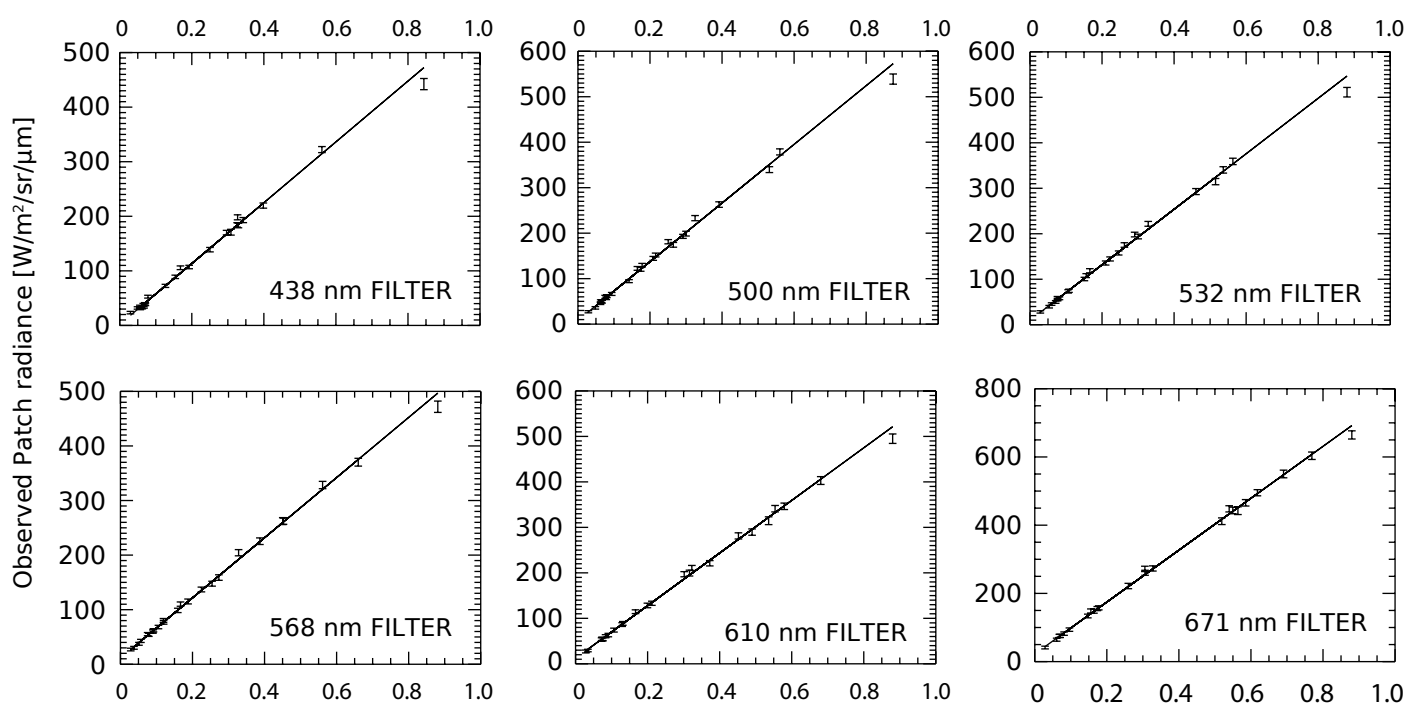

RWAC
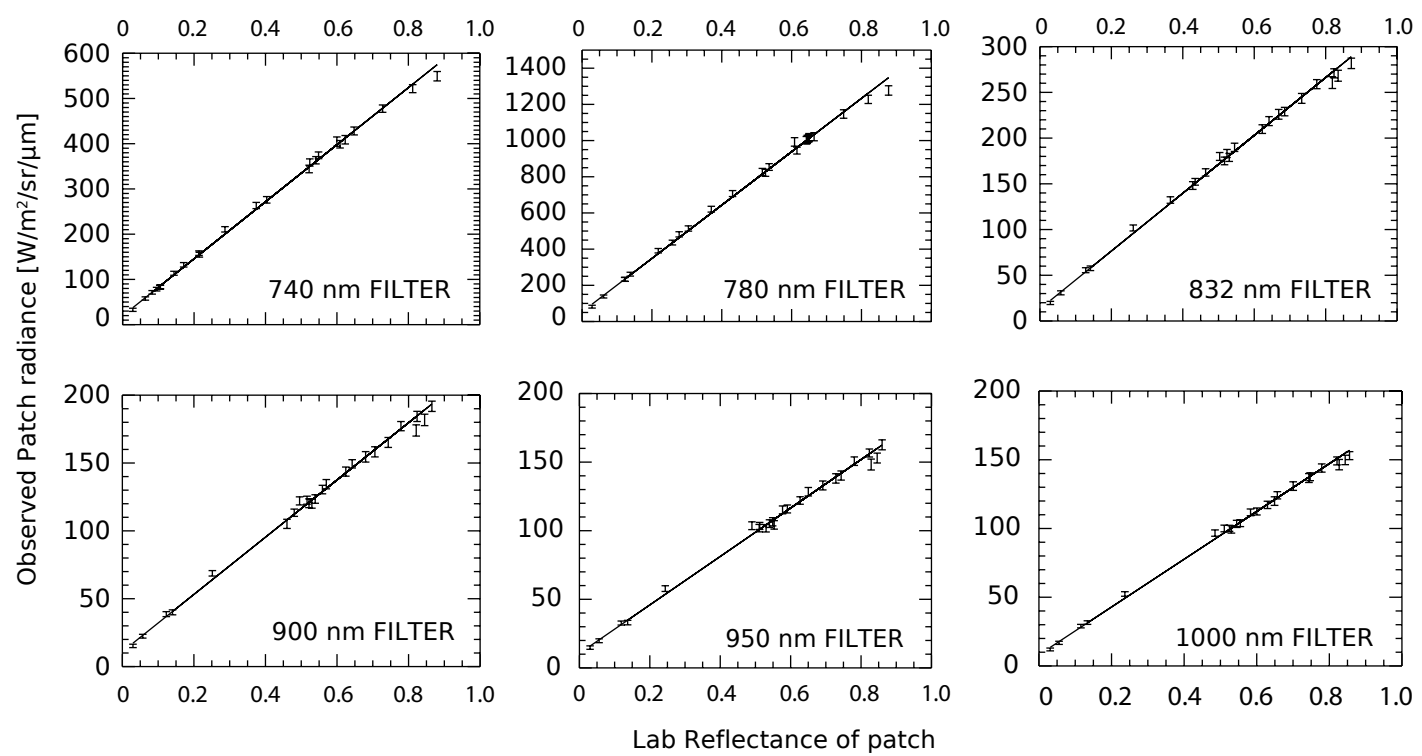

Fig 4 Plots of observed image radiance vs lab measured reflectance for each filter in LWAC and RWAC cameras. Illumination coefficients $m$ and $c$ are derived from the line of best fit between these two datasets as described in section 3.2.4. Points on this graph represent the observed vs measured values for each square of the 24 calibration target ROIs, bars represent their standard deviations. Note that if images have also been corrected for bias and dark signals, the straight line should pass through the origin as a signal of zero should be obtained for zero reflectance. ExoSpec does not perform dark and bias subtraction, as this will be performed after image downlink at the ROCC, thus we do not force the intercept to zero. 

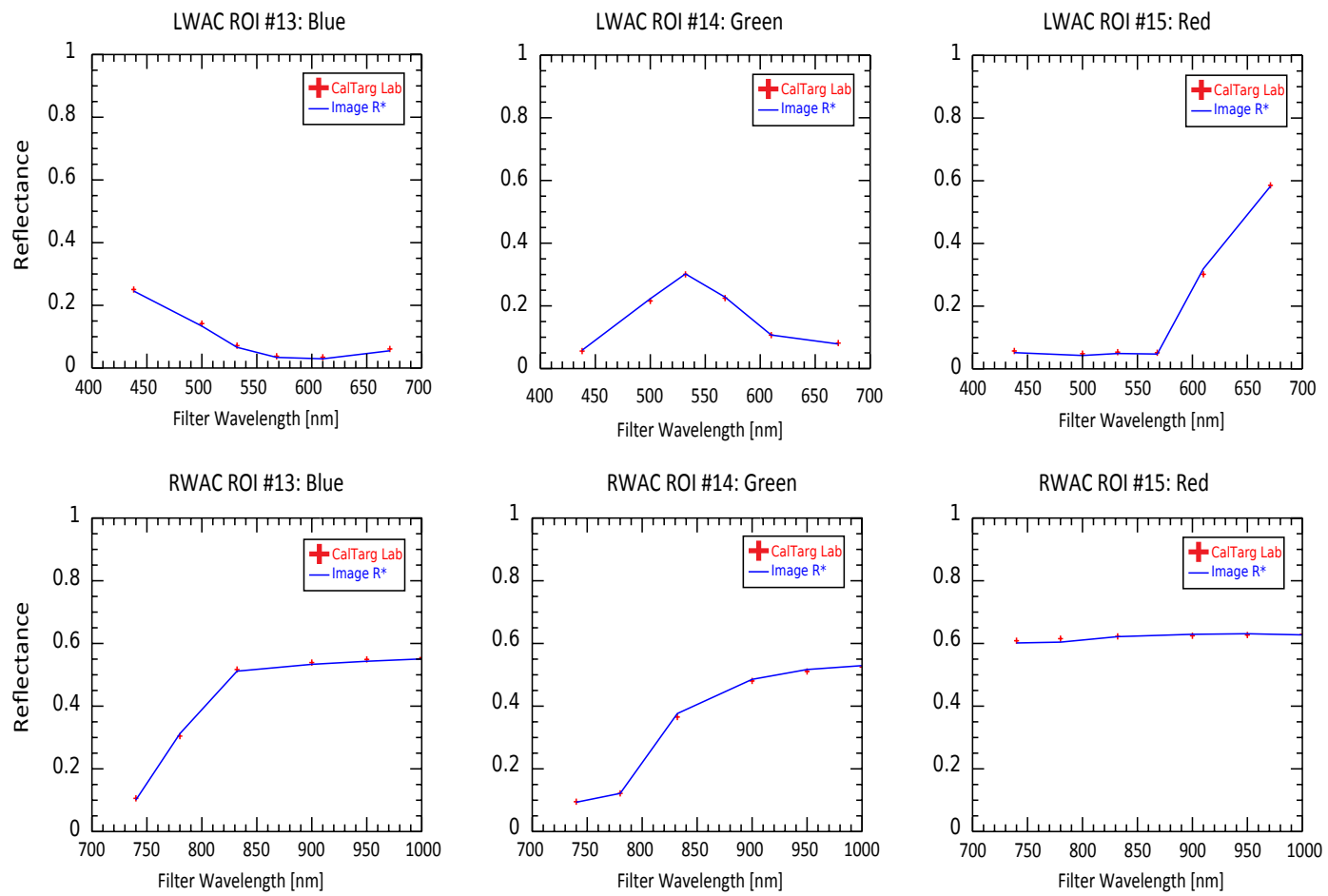

Fig 5 Plots of observed calibration target $R^{*}$ reflectance (blue) vs lab measured reflectance (red) across each filter for ROIs 13, 14, and 15 (Blue, Green, Red, as in Fig. 2) in LWAC and RWAC cameras, illustrating the agreement between calculated $\mathrm{R}^{*}$ and lab measured reflectance values. Two plots are produced per single ROI (one LWAC and one RWAC), resulting in 48 plots in total. 


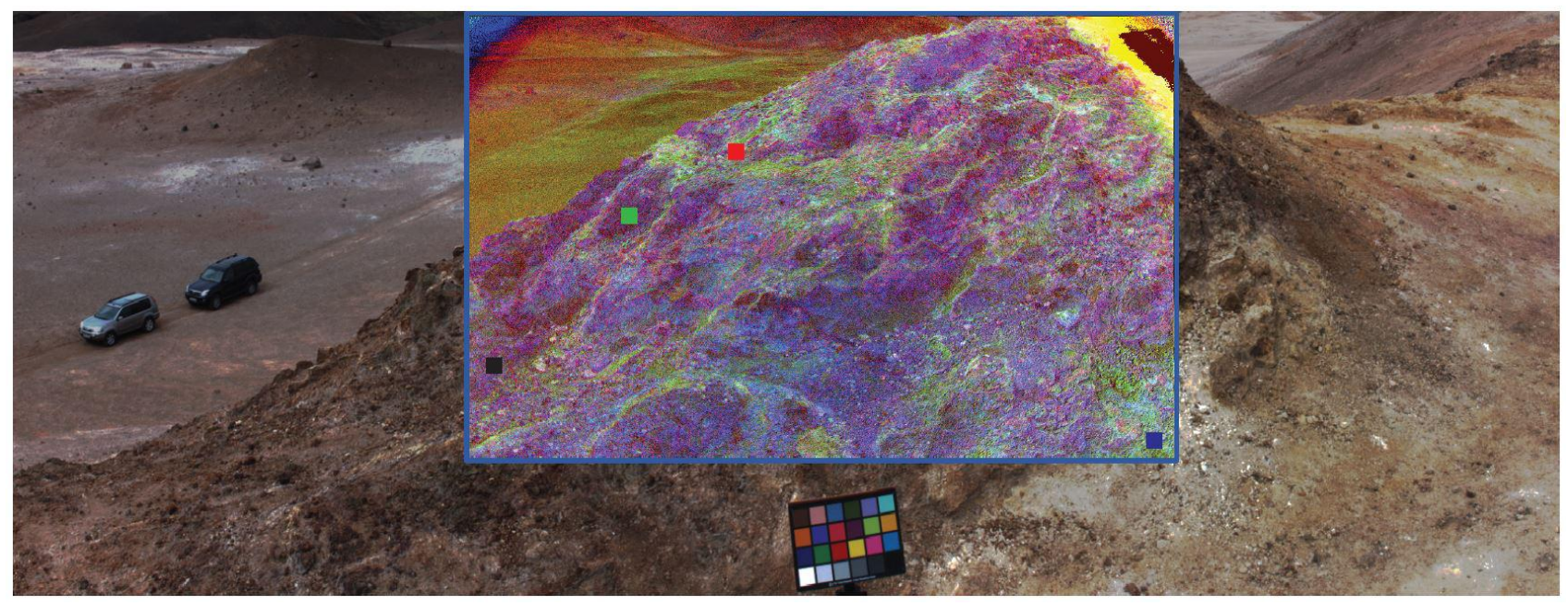

Fig 6 An RGB visible panorama of the altered pillow at the summit of Námafjall ridge from Fig. 3. Overlaid in the centre is an LWAC spectral parameter image made up of BD532, S532_610, R671_438 as RGB respectively (see Table 2 for reference). With this combination, altered gypsum veins clearly stand out from the basaltic pillow deposit in light green. Regions of Interest (ROI) in this image show locations of spectral sampling from AUPE3 and ISEM-E whose spectra are shown in Fig. 7. Gypsum veins also possess the most recognisable ISEM-E alteration signatures. Combining CLUPI-E fine scale information about individual veins could help to determine crystal size and whether they contain any additional minor phases or captured material.
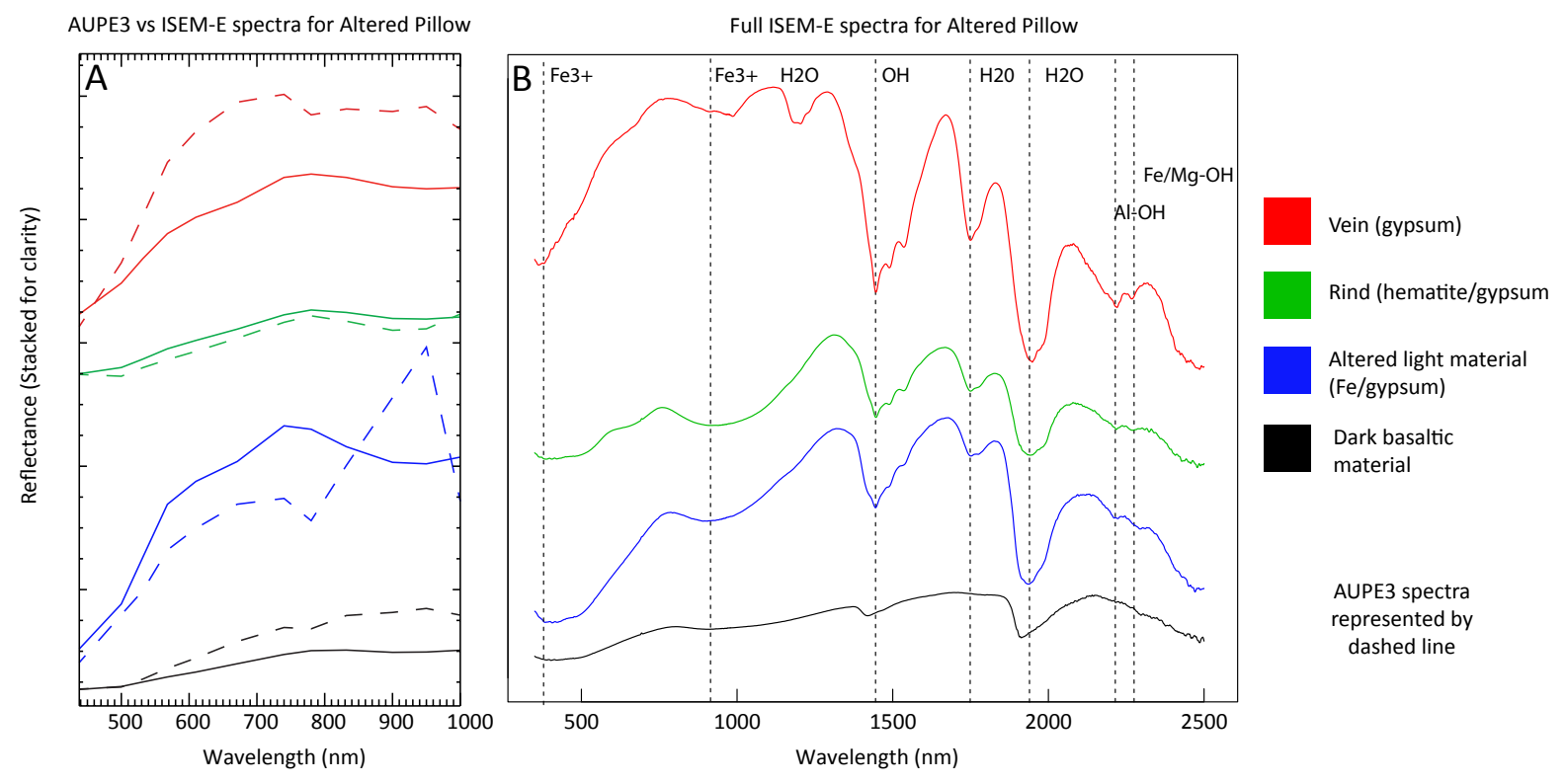

Fig 7 A: AUPE3 merged LWAC and RWAC spectra (extracted from their respective processed R* images from locations shown in Fig. 6) compared with ISEM-E spectra resampled to AUPE3 filter wavelengths. AUPE3 spectra are represented by dashed lines. B: Full ISEM-E spectra detailing additional band depths of interest. 


\subsection{Hanksville, Utah}

Fieldwork was also undertaken in Southern Utah, USA, near the town of Hanksville $\left(38.37^{\circ} \mathrm{N}, 110.71^{\circ} \mathrm{W}\right)$ and the site of the Mars Utah Rover Field Investigation (MURFI). ${ }^{28}$ In this region, alluvial deposits are preserved as inverted channel features in the Brushy Basin and Salt Wash Members of the Jurassic Morrison Formation, and the Ruby Ranch member of the Cretaceous Cedar Mountain Formation. These channel features preserve a record of flowing water on the surface. Fig. 8-A shows an RGB AUPE3 true colour panorama of the field site, an inverted channel feature consisting of a dark, oxidised base layer, through which exposed veins can be seen; silt, mud, and clay can be seen throughout this layer in varying oxidation states, and a very clear red to green mudstone/clay layer divides this feature. The channel is topped by a coarse to granular, and very poorly sorted fluvial channel sandstone layer, and a fine sandstone cap.

After processing this scene with ExoSpec (in the same manner as in section 4.1) Fig. 8-B shows mosaicked spectral parameter maps of the centre of the channel feature. These AUPE multispectral images clearly differentiate between the uppermost clay layer (shown in magenta) and an Fe-rich layer shown in green and blue.
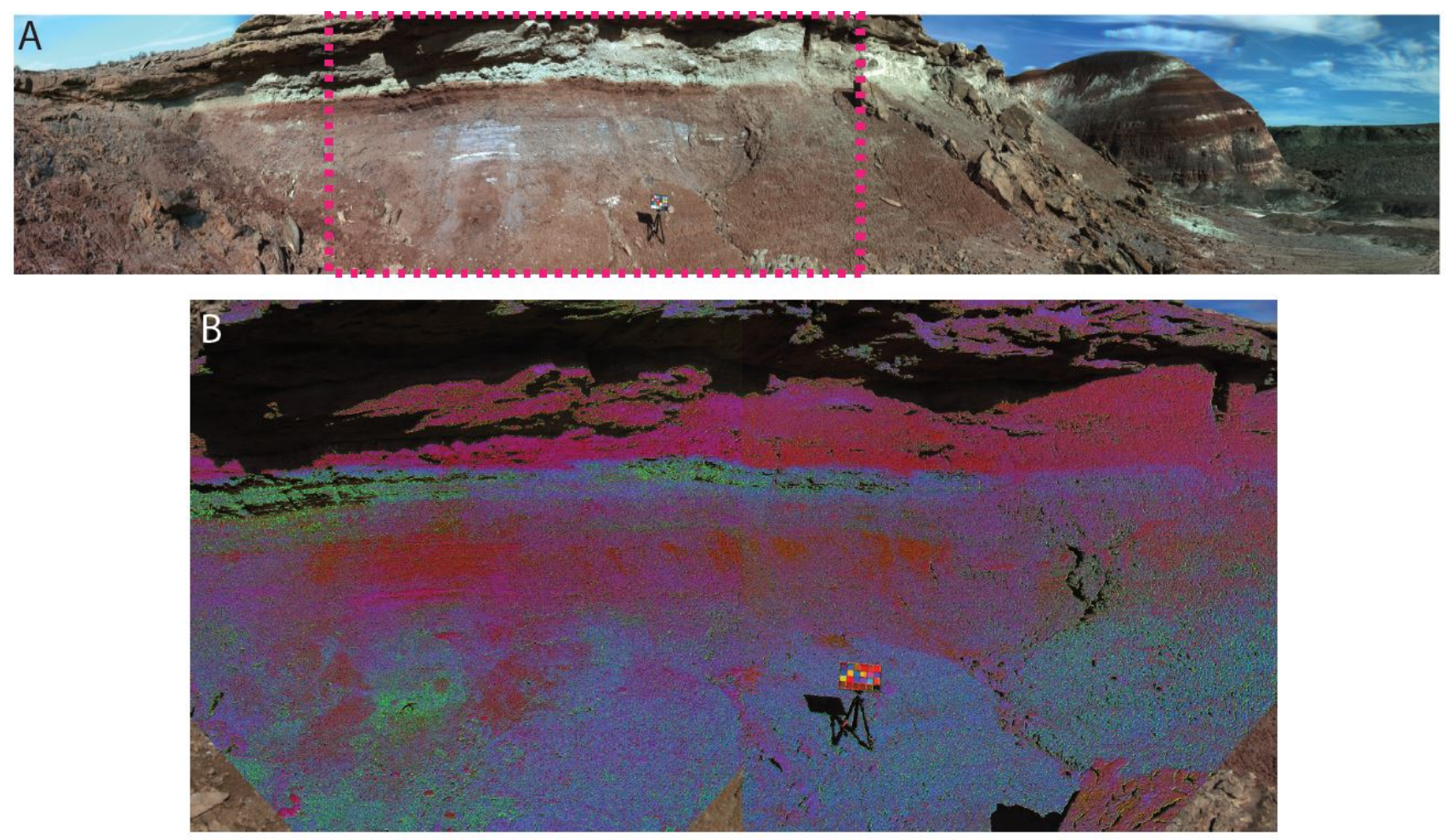

Fig 8 A: An RGB AUPE panorama showing the alluvial channel feature under study in Hanksville, USA. B: Spectral parameter map mosaic of the region marked with a dashed line in A. The band assignment for this LWAC multispectral image is R: S438_671, G: BD532, B: BD610. Due to this combination of band assignments, bright red regions in this image indicate less oxidation or a lack of dust presence. Green regions are indicative of Fe3+ containing minerals, particularly hematite, blue regions are also indicative of ferric materials, and can indicate goethite development due to an oxidisation state change from $\mathrm{Fe}^{2+}$ to $\mathrm{Fe}^{3+}$ ) within this wavelength region 


\section{Future development}

At its current stage of development, ExoSpec is an effective tool to streamline the processing of AUPE3 image data. There are many future add-ons to be incorporated into the program before the launch of the ExoMars 2020 rover mission, these will be discussed in this section.

ExoSpec does not leverage the stereo capabilities of AUPE3 (and therefore, PanCam). Thus, there is great potential to use output from Pro3D ${ }^{6}$ in tandem with ExoSpec, as there are several ways in which the process of spectral image analysis described in previous sections will benefit from extending the toolkit to incorporate the camera and scene geometry that can be extracted from stereo data. Ref. 6 demonstrates the advantages the combination of camera and scene geometry from Pro3D have for the structural analysis of geological outcrops. The ability to overlay multispectral information over accurate digital terrain models in combination with such structural geological information would prove valuable for strategic and tactical decision making.

Presently, spectra in the visible and near-infrared are matched between LWAC and RWAC via merging of spectra from manually created ROIs. By producing disparity maps via image rectification using CAHVOR ${ }^{29}$ camera model coefficients, the visible and near-infrared spectra of corresponding pixels can be stitched together to produce complete 12-band image cube products, akin to the Multispectral Data QUBs developed as MER Pancam data products. ${ }^{30}$ By incorporating this process into the ExoSpec toolkit we will be able to assess scene spectral variability simultaneously across all spectral and spatial samples, allowing for comprehensive unsupervised classification schemes to be deployed.

From the disparity maps produced above, or using output from Pro3D, photogrammetric depth maps can also be produced to map pixel coordinates to 3D world space, consequently surface normals can be fitted to rock facets. By incorporating surface normal corrections into the $\mathrm{R} *$ reflectance conversion, along with knowledge of camera pose, solar azimuth, and elevation, spectra can be normalised for closer correspondence to laboratory spectra. This would allow ExoSpec to perform photometric studies, which have helped to constrain the physical and mineralogical properties of rock and soil classes at previous landing sites, most notably at Gusev Crater ${ }^{31}$ and Meridiani Planum. ${ }^{32}$

In future versions of ExoSpec, Bidirectional Reflectance Distribution Functions (BDRF) will be used in place of a single $\mathrm{R}^{*}$ reflectance value in order to take solar incidence and detector angles into account for each scene being analysed. To this end, a Look Up Table (LUT) will be built into ExoSpec and used to select the appropriate lab reflectance measurement for the calibration target and minimise offset between observed image and laboratory measurements. To facilitate this, the BDRF of each of the calibration target squares will be measured for each AUPE3 filter wavelength at Aberystwyth University. Provided we know the sun's azimuth and elevation when an image is captured, we can process the observed reflectance using the appropriate lab reflectance values for each of the calibration target squares using the LUT. ${ }^{33}$

ExoSpec does not yet have the capability to perform spectral matching of AUPE3 spectra with higher spectral resolution spectra obtained from ISEM-E. AUPE3 spectra manually extracted from ROIs within an image can be used to compare with ISEM-E spectra of the same regions taken in the field to ensure cross-calibration of the two instruments. In the case of PanCam and ISEM, whose wavelength ranges do not overlap, the cross-calibration results from their emulators will prove useful when comparing data from these two instruments. Future functionality may be added for the unmixing of ISEM footprints using CLUPI or HRC images. Using image segmentation, CLUPI and HRC images 
may facilitate the estimation of endmember spectra and their abundances contributing to an ISEM footprint.

In section 3.2.5 the uncertainty values of the illumination coefficients $m$ and $c$ were calculated by ExoSpec. Pixel-by-pixel calculation of the total uncertainty in $\mathrm{R}^{*}$ (a combination of the combined uncertainties from $m, c$, and $S$ in Eq. 2) will also be calculated in future versions of the toolkit.

At present, ExoSpec calculates all summary parameters given in Table 2 and assigns them equal importance. Given time and data volume constraints, it will be important to determine which spectral parameters best capture the spectral variability within an image, particularly if only a subset of all available filters are able to be used for imaging in one sol. We are currently investigating optimal combinations of these and newly developed parameters, and buttons for the calculation of spectral parameters based on available filters will be included in ExoSpec.

Developing a multi-purpose toolkit to perform the above studies at this stage of instrument development allows for its methods to be tested prior to mission launch through analogue studies. This will allow for the methods to be objectively classified into tactical and strategic techniques, maximising both the efficiency and information extraction during time-critical sol-by-sol rover planning.

\section{Conclusion}

This paper provides a snapshot into the development, capabilities, and current status of ExoSpec. ExoSpec will continue to be developed as the launch for ExoMars 2020 approaches, greater functionality will be incorporated to facilitate analysis alongside with ISEM and CLUPI instruments. Through analogue studies undertaken in Iceland and the USA, we demonstrate that ExoSpec performs flat-fielding, radiometric correction, calculation of illumination coefficients, radiance to reflectance conversion, and science product generation, facilitating the interpretation of entire multispectral scenes. It is currently being tested with AUPE3 images, but PanCam processing functionality will be added alongside the AUPE3 processing menu as further calibration parameters become available for the PanCam instrument.

\section{Acknowledgments}

This work was supported by the UK Space Agency (ST/P001297/1 and ST/P001394/1). Cousins also acknowledges the Royal Society of Edinburgh for funding. The authors would also like to thank Mark Fox-Powell, Arola Moreras Marti, and Aubrey Zerkle for field assistance in Iceland; and Robert Barnes, Matthew Balme, Christy Caudill, Joel Davis, and Peter Fawdon for their assistance in Utah.

\section{References}

1 D. Barnes, E. Battistelli, R. Bertrand, et al., "The ExoMars rover and Pasteur payload Phase A study: an approach to experimental astrobiology," International Journal of Astrobiology 5(3), 221-241 (2006).

2 J. L. Vago, F. Westall, Pasteur Instrument Teams, et al., "Habitability on early Mars and the search for biosignatures with the ExoMars rover," Astrobiology 17(6-7), 471-510 (2017).

3 A. Coates, R. Jaumann, A. Griffiths, et al., "The PanCam instrument for the ExoMars rover," Astrobiology 17(6-7), 511-541 (2017). 
4 O. I. Korablev, Y. Dobrolensky, N. Evdokimova, et al., "Infrared spectrometer for ExoMars: a mast-mounted instrument for the rover," Astrobiology 17(6-7), 542-564 (2017). PMID: 28731817.

5 J.-L. Josset, F. Westall, B. A. Hofmann, et al., "The Close-Up Imager onboard the ESA ExoMars rover: objectives, description, operations, and science validation activities," Astrobiology 17(67), 595-611 (2017). PMID: 28731819.

6 R. Barnes, S. Gupta, C. Traxler, et al., "Geological analysis of martian rover-derived digital outcrop models using the 3-D visualization tool, Planetary Robotics 3-D ViewerPRo3D," Earth and Space Science (2018).

7 M. P. Golombek, R. C. Anderson, J. R. Barnes, et al., "Overview of the Mars Pathfinder Mission: Launch through landing, surface operations, data sets, and science results," Journal of Geophysical Research: Planets 104(E4), 8523-8553 (1999).

8 S. K. LaVoie, W. B. Green, A. J. Runkle, et al., "Processing and analysis of Mars Pathfinder science data at the Jet Propulsion Laboratory's science data processing systems section ," Journal of Geophysical Research 104(E4), 8831-8852 (1999).

9 J. Bell, J. Joseph, and J. Sohl-Dickstein, MER Pancam data processing user's guide. Cornell University, 1.0 ed. (2004).

10 J. F. Bell, A. Godber, S. McNair, et al., "The Mars Science Laboratory Curiosity rover Mastcam instruments: Preflight and in-flight calibration, validation, and data archiving," Earth and Space Science 4(7), 396-452 (2017).

11 J. Harris, C. Cousins, M. Gunn, et al., "Remote detection of past habitability at Mars-analogue hydrothermal alteration terrains using an ExoMars Panoramic Camera emulator," Icarus 252, $284-300$ (2015).

12 S. Murchie, R. Arvidson, P. Bedini, et al., "Compact Reconnaissance Imaging Spectrometer for Mars (CRISM) on Mars Reconnaissance Orbiter (MRO)," Journal of Geophysical Research: Planets 112(E05S03) (2007).

13 A. J. Runkle, J. J. Lorre, D. A. Alexander, et al., "The Mars Pathfinder Science Data Processing System." (1998).

14 D. A. Alexander, R. G. Deen, P. M. Andres, et al., "Processing of Mars Exploration Rover imagery for science and operations planning," Journal of Geophysical Research 111(E02S02) (2006).

15 W. Farrand, J. Bell, J. Johnson, et al., "Spectral variability among rocks in visible and nearinfrared multispectral Pancam data collected at Gusev crater: Examinations using spectral mixture analysis and related techniques," Journal of Geophysical Research 111(E02S15) (2006).

16 W. H. Farrand, J. F. Bell, J. R. Johnson, et al., "Visible and near-infrared multispectral analysis of rocks at Meridiani Planum, Mars, by the Mars Exploration Rover Opportunity," Journal of Geophysical Research 112(E06S02) (2007).

17 S. M. Pelkey, J. F. Mustard, S. Murchie, et al., "CRISM multispectral summary products: Parameterizing mineral diversity on Mars from reflectance," Journal of Geophysical Research: Planets 112(E8) (2007).

18 W. H. Farrand, J. F. Bell, J. R. Johnson, et al., "Rock spectral classes observed by the Spirit Rovers Pancam on the Gusev Crater Plains and in the Columbia Hills," Journal of Geophysical Research 113(E12S38) (2008). 
19 C. E. VivianoBeck, F. P. Seelos, S. L. Murchie, et al., "Revised CRISM spectral parameters and summary products based on the currently detected mineral diversity on Mars," Journal of Geophysical Research: Planets 119(6), 1403-1431 (2014).

20 M. Morgan, F. Seelos, and S. Murchie, "The CRISM Analysis Toolkit (CAT): Overview and Recent Updates," in Third Planetary Data Workshop and The Planetary Geologic Mappers Annual Meeting, LPI Contributions 1986, 7121 (2017).

21 C. Cousins, M. Gunn, B. Prosser, et al., "Selecting the geology filter wavelengths for the ExoMars Panoramic Camera instrument," Planetary and Space Science 71, 80-100 (2012).

22 A. Griffiths, A. Coates, J.-L. Josset, et al., "The Beagle 2 stereo camera system: scientific objectives and design characteristics," Planetary and Space Science 53, 1446-1482 (2005).

23 J. F. Bell, S. W. Squyres, K. E. Herkenhoff, et al., "Mars Exploration Rover Athena Panoramic Camera (Pancam) investigation," Journal of Geophysical Research: Planets 108(E12) (2003).

24 D. Barnes, M. Wilding, M. Gunn, et al., "The PanCam Calibration Target (PCT) and multispectral image processing for the ExoMars 2018 mission," in EPSC-DPS Joint Meeting 2011, 289 (2011).

25 J. F. Bell, J. Joseph, J. N. SohlDickstein, et al., "Inflight calibration and performance of the Mars Exploration Rover Panoramic Camera (Pancam) instruments," Journal of Geophysical Research: Planets 111(E2) (2006).

26 M. Gunn, Spectral imaging for Mars exploration. PhD thesis, Aberystwyth University (2013).

27 R. J. Reid, P. H. Smith, M. Lemmon, et al., "Imager for Mars Pathfinder (IMP) image calibration," Journal of Geophysical Research: Planets 104(E4), 8907-8925 (1999).

28 M. Balme, M. Curtis-Rouse, S. Banham, et al., "UK Space Agency Mars Utah Rover Field Investigation 2016 (MURFI 2016): overview of mission, aims and progress," in Lunar and Planetary Science Conference, (2017). Abstract 1837.

29 K. Di and R. Li, "CAHVOR camera model and its photogrammetric conversion for planetary applications," Journal of Geophysical Research: Planets 109(E4) (20004).

30 J. M. Soderblom, J. F. Bell, R. E. Arvidson, et al., "Mars Exploration Rover Pancam photometric data QUBs: definition and example uses," AGU Fall Meeting Abstracts , P21A-0198 (2004).

31 J. R. Johnson, W. M. Grundy, M. T. Lemmon, et al., "Spectrophotometric properties of materials observed by Pancam on the Mars Exploration Rovers: 1. Spirit," Journal of Geophysical Research: Planets 111(E2) (2006).

32 J. R. Johnson, W. M. Grundy, M. T. Lemmon, et al., "Spectrophotometric properties of materials observed by Pancam on the Mars Exploration Rovers: 2. Opportunity," Journal of Geophysical Research: Planets 111(E12) (2006).

33 D. Barnes, M. Wilding, M. Gunn, et al., "Multi-spectral vision processing for the ExoMars 2018 mission," in 11th Symposium on Advanced Space Technologies in Robotic and Automation (ASTRA 2012), (2011). 